\section{Technology to support integrated Antimicrobial Stewardship Programs: a user centered and stakeholder driven development approach}

\author{
Nienke Beerlage-de Jong, ${ }^{1}$ \\ Lisette van Gemert-Pijnen, ${ }^{1}$ \\ Jobke Wentzel, ${ }^{2}$ Ron Hendrix, ${ }^{3,4}$ \\ Liseth Siemons ${ }^{1}$
}

${ }^{1}$ Center for eHealth and Wellbeing

Research, Department of Psychology,

Health and Technology, University of

Twente, Enschede; ${ }^{2}$ Department of

Media, Communication and

Organization, University of Twente,

Enschede; ${ }^{3}$ Clinical Bacteriology Unit,

Department of Medical Microbiology,

University of Groningen, University

Medical Center Groningen; ${ }^{4}$ Certe

Laboratory for Infectious Diseases,

Groningen, the Netherlands

\begin{abstract}
The rise of antimicrobial resistance (AMR) is a severe global health problem. Tackling this problem requires the prudent prescribing of antimicrobials. This is promoted through Antimicrobial Stewardship Programs (ASPs). In this position paper we describe i) how a socio-technical multidisciplinary approach (based on the CeHRes Roadmap) can be applied in the development and implementation of Antimicrobial Stewardship technologies and ii) how this approach can be of value to support Antimicrobial Stewardship in practice. The CeHRes Roadmap entails five different phases to explore and test how an eHealth technology can be tailored to the target group and successfully implemented in practice: i) contextual inquiry, ii) value specification, iii) design, iv) operationalization, v) evaluation. In this position paper we describe the lessons learned from research and practice to guide future developments of technology based ASP interventions. Since AMR is a huge wicked problem on a global level, it requires innovative methods and models to empower general public and professionals to be proactive rather than reactive in a digitalized world. We highlight how to combat the dangerous rise of antimicrobial resistance in the future.
\end{abstract}

\section{Introduction}

The rise of antimicrobial resistance (AMR) is a severe problem, ${ }^{1,2}$ a problem that requires a sustainable program and incentives to implement safe care in practice. Such programs are launched as Antimicrobial Stewardship Programs (ASPs). These programs aim to monitor infections, implement safe work strategies, prevent the misuse of antimicrobials and educate healthcare workers. Although they are promising, they do not meet their users' needs and work practices. Due to many factors, such as a lack of incentives, bad hygiene behaviors, unworkable regulations, lack of knowledge, and misuse of antimicrobials, our healthcare system becomes a breeding ground for antimicrobial resistance. ${ }^{2}$ The mere fact that, in the USA alone over two million people become infected with multidrug resistant organisms each year, resulting in 23.000 deaths annually, and that costs of antimicrobial resistance are estimated at $\$ 20$ billion a year (USA), stresses the necessity to take action. ${ }^{3,4}$

Antimicrobial resistance is a huge wicked problem on a global level, ${ }^{5}$ with no single solution that could by itself solve the problem, requiring a so called theragnostic model based on infection prevention, antibiotic and diagnostic stewardship programs. ${ }^{6}$ This multidisciplinary stewardship approach requires intensive collaboration among stakeholders. 5,7 The current state-ofthe-art is to not only combat superbugs with the development of new drugs, but also to find behavioral solutions in social sciences to create optimal conditions for the stewardship programs. The Centers for Disease Control and Prevention also stated that stewardship programs require a coordinated multidisciplinary approach and the continuous tracking of prescribing behaviors and resistance patterns, as well as information and education strategies to support healthcare workers (HCWs). ${ }^{3}$ This is where technology based behavioral solutions will help. With the introduction of technology in stewardship programs infection prevention, antibiotic prescribing and diagnostic interventions can be personalized to the needs of patients and healthcare workers. ${ }^{6}$

In this position paper we describe several eHealth technologies that we developed to support infection prevention and/or ASP. The aim of this paper is to look back on and provide an overview of our years of experience with a research-based approach of developing user-centered eHealth technology (following the CeHRes Roadmap) in the field of ASPs, and to demonstrate why this approach has value for technologies that support ASPs. It is not within the scope of this paper to present measurement data of
Correspondence: Nienke Beerlage-de Jong, Center for eHealth and Wellbeing Research, P.O. Box 217, 7500AE Enschede, the Netherlands,

Tel.: +31.53.4893517.

E-mail: n.beerlage-dejong@utwente.nl

Key words: Antimicrobial Resistance; Antimicrobial Stewardship Programs; eHealth, CeHRes Roadmap.

Acknowledgements: The authors would like to thank all healthcare workers who participated in our research.

Contributions: the authors contributed equally.

Conflict of interest: the authors declare no potential conflict of interest.

Funding: Most of the research and technologies that are described in this paper were supported by the INTERREG IVa EurSafety Health-net project.

Received for publication: 9 August 2016.

Revision received: 26 January 2017.

Accepted for publication: 30 January 2017

This work is licensed under a Creative Commons Attribution-NonCommercial 4.0 International License (CC BY-NC 4.0).

(C) Copyright N. Beerlage-de Jong et al., 2017 Licensee PAGEPress, Italy

Infectious Disease Reports 2017; 9:6829

doi:10.4081/idr.2017.6829

the clinical applicability, impact or effectiveness of the technologies. The CeHRes Roadmap offers a guideline that is used to develop ASPs that fit its users and its context. In describing how it can be applied to the development of technology to support ASPs, we hope to offer guidance to eHealth technology developers so that such technologies actually work, help and are implementable. To do so, we describe i) how a socio-technical multidisciplinary approach (based on the CeHRes Roadmap) can be applied in the development and implementation of Antimicrobial Stewardship technologies and ii) how this approach can be of value to support Antimicrobial Stewardship in practice. We describe the lessons learned from research and practice, to guide future developments of tech-based ASP technologies. In doing so, we elaborate on the report by the Centers for Disease Control and Prevention, which describes what interventions should be implemented in an ASP. ${ }^{3}$ We complement this knowledge with insight in how to realize such interventions, through technology. 


\section{The CeHRes Roadmap, a multidisciplinary development approach}

The CeHRes Roadmap was developed at the Centre for eHealth and Wellbeing Research of the University of Twente and introduced in the development of technologies for infection prevention and control in several projects (www.eursafety.eu; mrsanet.nl). ${ }^{8}$ The Roadmap prioritizes the human standard in the development of technologies for the improvement of healthcare, incorporating user-centered design principles, persuasive technology, and a stakeholder value development approach (business modeling). The integration of a usercentered design approach with a stakeholder-driven collaboration procedure during development enables a sustainable implementation of technologies in healthcare. In our view, the development of such technologies is more than creating a vehicle or tool to support people. It is a process of cocreation with end-users and other stakeholders to guarantee a fit between technology, users, and context (way of living and working). This co-creation process is coupled with continuous and iterative cycles of evaluation (formative evaluation) to gain insight in whether a technology can contribute to improving healthcare and what barriers hinder its implementation. Therefore, implementation is not a post design step, but is interwoven with the development of a technology. As such, eHealth development is not an ad hoc activity, but a structural approach to set the conditions for personalized healthcare using technology.

The Roadmap entails five different phases to explore and test how eHealth technology can be perfectly personalized and tailored to the target group and successfully implemented in practice (Figure 1)

\section{Contextual inquiry}

The objective of the contextual inquiry phase is to identify the weak and strong points of the current situation, for example current Stewardship approaches to reduce AMR, to inquire the needs of stakeholders to improve health, how technology can be used to deal with the problems and needs, and to identify regulations and conditions that should be taken into account (i.e., legislation, regulations, policy) to implement an eHealth technology. ${ }^{9}$

\section{Value specification}

The value specification phase builds on the results of the contextual inquiry. The identified key-stakeholders determine the added values (economical, medical, organizational etc.) that they want to achieve by means of a technical solution for the improvements in healthcare, such as a reduction of AMR. These values can be ranked in order to create a feasible technology and to set conditions for implementation (see also www.eHealthWiki.org). ${ }^{10}$ The values will be translated by the project management team (Research \& Development) into system, content and service requirements in a value map.

\section{Design}

Within this phase of the Roadmap, the design (the look-and-feel) of the technology is developed. In close cooperation with endusers (e.g., doctors, nurses, patients) several design-prototypes (from paper-based until fully functioning technology) can be developed and tested using scenarios that represent the context: the use of an AMR technology in healthcare practice. For the design of such prototypes, usability principles and persuasive design models (e.g., the Persuasive Systems Design Model) can be used to ensure a fit between technology, its use context and its users. ${ }^{9,11,12}$

\section{Operationalization}

This phase entails activities to introduce a technology in healthcare. An implementation and a business model (e.g., timing, activities, actors, budget) is developed together with an evaluation plan to examine whether the goals, or values are realized. ${ }^{7}$

\section{Evaluation}

To examine whether an eHealth technology works and helps, ongoing evaluation rounds (i.e., formative evaluation) are conducted throughout the whole developmental phase. Additionally, a final evaluation round (i.e., summative evaluation) is performed after implementation to measure the effects on behavior change, attitudes and the impact on the goals to be realized.

The CeHRes Roadmap is a guide that can be used integral to develop and implement technologies, as a guide to assess the developmental process or to guide a (partial) redesign process

\section{Development of technology for antimicrobial stewardship pro- grams via the CeHRes Roadmap}

In this paper, we describe how the CeHRes Roadmap was used to develop several technologies to share information and to communicate with HCWs, about how to prevent infections and how to avoid antimicrobial resistance. The CeHRes Roadmap has a good fit with complex problems like AMR and the integrated stewardship model because of its' multidisciplinary and socio technical based development approach. ${ }^{6} \mathrm{We}$ elaborate per ASP technology on a specific phase of the Roadmap, most suitable to deal with wicked problems and to focus on stewardship supporting HCWs with technologies relevant for AMR. The described technologies are or will all be offered through and bundled on a website called Infection Manager (beta version available on www.infectionmanager.com), which serves as a platform for communication and information sharing to support Integrated AMR Stewardship. In this paper we describe i) a technology to support decision making about antimicrobials (focusing on Contextual Inquiry), ii) a maturity model to guide the implementation of ASP technologies (Value specification \& Operationalization), iii) a technology to support nurses in antimicrobial administration (Design to fit the use context), and d) a technology to register infections for nursing homes (Persuasive design).

\section{Technology to support decision making about antimicrobials (contextual inquiry)}

\section{Background}

Decision making in an ASP is a complex matter. Many factors influence a decision on how to diagnose and decide what treatment a patient needs. To support physicians in making decisions about the prescribing of antimicrobials, Clinical Decision Support Systems (CDSSs) are proposed and often implemented. ${ }^{13}$ However, their success largely differs, mainly due to low use and low adherence rates. To increase the chances of success, CDSSs should fit its users' needs and expectations, but also their work processes.

\section{Activities}

We conducted a Contextual Inquiry to identify why and how a clinical decision system can support the prescribing of antimicrobials. A scoping review of the literature on existing CDSSs provided insight in their current development, design and implementation. It revealed a lack of involvement of potential end-users during the development, but also a lack of attention for the design process of CDSSs (most publications do not report on how a CDSS was developed). In general, existing CDSSs mainly have a clinical focus, but lack attention for stewardship and how to support decisions in practice. Insights from the scoping review were used to develop, test, 
and evaluate scenario-based prototypes with end users. Observing physicians while they interact with these prototypes provided insight into the existing work processes (e.g., current information sources), problems that end-users experience (e.g., indistinctness about duration of an AB therapy) and users' preferences and needs regarding the look and feel of the technology.

\section{Lessons learned}

Early involvement of stakeholders and end-users is necessary to develop user friendly ASP technologies. Stakeholders, like experts in the field of infections and in other medical specialties, are needed to continuously keep an eye on the fit between technology and clinical practice, and to set conditions for implementation.

Scenario-based prototype evaluations enable potential end-users to visualize and verbalize their work processes and problems they encounter, and to think outside their comfort zone, to discuss opportunities of technology based ASP.

Scenario-based prototype evaluations (and cross-referencing its findings among other stakeholders) provide valuable information to harmonize and translate medically focused protocols and guidelines into practice based instructions.

Involvement of end-users provides clear insight into improvements for the complex work environment that healthcare is. For example, currently most eHealth technology to support ASPs is directional, restrictive and less integrated with practice. Medical autonomy (which is a prerequisite for being able to deal with complex and ever changing circumstances) of e.g., physicians is sometimes neglected in ASP. End-users clearly indicated a need for a technology to respect and keep account with their medical autonomy, and to give room for deliberation (i.e., diagnostics as triage for decision making).

\section{Maturity model to implement antimicrobial stewardship technologies (value specification and operationalization)}

\section{Background}

Infection prevention and control should be considered as an integrated healthcare approach. ${ }^{6}$ To determine what ASP interventions should be developed and to facilitate implementation, involvement of stakeholders is crucial. A maturity model can help to assess the activities already done and to guide the development of interventions, in cooperation with stakeholders such as medical professionals, infection experts, and (local) managers.

\section{Activities}

Business modeling activities were performed to identify the key stakeholders and to determine the values that have to be realized with ASPs in practice. ${ }^{14}$ Multidisciplinary focus groups were conducted to decide what ASP technologies should be developed, to define their requirements, and to identify necessary resources and skills, from planning until operationalization of an ASP. ${ }^{15}$

\section{Lessons learned}

ASP interventions, supported by technology, can be bundled in a toolkit. Such ASP toolkit offers a set of technologies that are intended to support an integrated ASP approach; ${ }^{16}$

i. Information app to support antimicrobial prescribing (aimed at physicians)

ii. Interactive antimicrobial prescription decision support app

iii. Information app to support antimicrobial administration (in the Netherlands aimed at nurses)

iv. Information app to transfer patient care

v. Tool to facilitate audits by the ASPteam

vi. Alerts/notifications

vii. Education via E-Learning

Our maturity assessment checklist with questions regarding the status nascendi of local technologies can be used to decide the maturity of the ASP initiatives (e.g., done, planned).

The maturity model that we developed guides the development of these technologies considering the aims, resources and capabilities of a local organization and (inter)national regulations for ASP. It presents the necessary information in manageable and relevant fragments to A-teams that are in the process of implementing ASP. That also makes it different from the few existing ASP guidelines or toolkits that exist. ${ }^{14}$
In Figure 2 an overview of the maturity model for implementing ASP is given. Each button will have submenus with further implementation information.

\section{Technology to support nurses in antimicrobial administration (design)}

\section{Background}

Optimal use of antimicrobials relies on careful prescription and administration. Often, the manuals and protocols that describe these complex activities are expert-driven, difficult to understand, inaccurate, or not in line with novel insights. ${ }^{17}$

Nurses can play a key role in administration and monitoring of antimicrobials. They can inform physicians on the (actual) use and effects of antimicrobials on their patients, based on their daily care activities. ${ }^{17}$ This informative role is implicit in medical protocols. Yet, the cooperation between nurses and physicians in streamlining antimicrobial care for patients is crucial for successful ASPs. ${ }^{18}$

\section{Activities}

The contextual inquiry that was carried out in the participating local teaching hospital indicated that nurses need information that supports them in their preparation, administration and monitoring tasks, and that assists them to observe possible risks or adverse events associated with antimicrobial therapy. ${ }^{17}$ User-centered design principles with a focus on nurses' daily tasks were used to design prototypes of the Antibiotic Information app with nurses, doctors, microbiologist and pharmacists. To change the protocols into more usable and task-oriented information sources, card sorting was done. This method helps to create intuitive information structure for online information sources. ${ }^{19}$ Nurses sorted cards with small chunks of information (e.g., parts of protocols, reference documents) in (what they regard as) logical groups. In addition, they

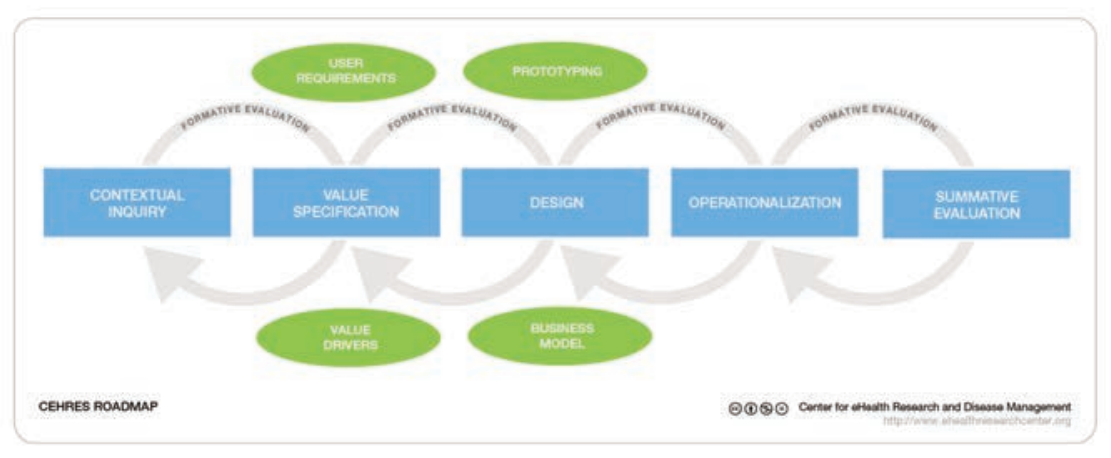

Figure 1. The CeHRes Roadmap. ${ }^{8}$ 
named each group while thinking aloud. On the resulting sorts, a cluster analysis was performed to show how different information parts are related, according to the nurses, based on their experience. The results show that the app should present information in a task-oriented way, including practical instructions (e.g., on dose, preparation, administration and monitoring). In addition, background information (e.g., about the disease that requires antimicrobial treatment or specific characteristics of the antimicrobials) should be included (Figure 3).

\section{Lessons learned}

To support the tasks of nurses, insight in information use is required.

For technologies to be optimally supportive, explicit elicitation of and decisions on functional requirements (i.e., what the technology should be able to do) and design requirements (i.e., what interfaces should look like, how information should be structured to support tasks) is needed.

Information should primarily be task oriented, but additional (in-depth) explanations and resources should be accessible as well, to reflect medical standards.

Information availability is a prerequisite for active nurse involvement in ASP, but eLearning is needed to stimulate novel practices.

\section{Technology to register infections in nursing homes (design)}

\section{Background}

Once a year, nursing homes that are part of the (Dutch) iPrevent network register all their clients in a prevalence measurement of nursing home-associated infections and related risk factors (e.g., presence of catheters, antimicrobial use). This task is performed by elderly care physicians, who thus have to register numerous clients within a short period. The previous (paperbased) tool that was used to do so was time consuming, laborious and prone to interpretation errors. ${ }^{11}$ To deal with these issues, a web-based registration and monitoring app for nursing home-associated infections (the Prevalence app) was developed.

To increase adherence it is important that technologies to support ASP, support its end-users in performing their tasks and should motivate them in doing so. Persuasive technology offers such support, ${ }^{12}$ to improve adherence with certain rules of conduct. Persuasive infection management is not focused on punishing bad compliance, as this has a demotivating effect, but on a positive, rewarding approach to behavioral change.

\section{Activities}

Scenario-based prototypes were evaluated by elderly care physicians (the intended end-users). For scenario-based user testing, a low-fidelity prototype of the Prevalence app was developed, incorporating elements of persuasive design. For example:

Primary Task Support was provided to simplify the tasks (e.g., reduction to shorten questions) and to make it less time consuming (e.g., tunnelling to omit irrelevant questions).

Dialogue Support was incorporated to avoid errors (e.g., suggestion to avoid typing errors, Figure 4) and to make sure all relevant information is included (e.g., reminders to add laboratory test results as soon as they are available).

System Credibility Support was embedded to ensure its face validity (e.g., by clearly displaying the logo of the INTERREG
IVa EurSafety Health-net project that it was developed in) and its reliability (e.g., by embedding the app in a larger website which provided additional information about the project and the experts who were involved in its development).

Social Support was used to allow nursing homes to reflect on their own infection rates (i.e., social Comparison to compare the own data that with a benchmark of the other participating nursing homes).

\section{Lessons learned}

Persuasive technology matters. Preliminary evaluations showed that endusers performed (fictional, but real-life) tasks faster and with less mistakes than with the prior web-based system. ${ }^{20}$

Preliminary evaluations indicated that the perceived persuasiveness of the Prevalence app was higher than of the prior system. $^{20}$

End-users expressed appreciation for
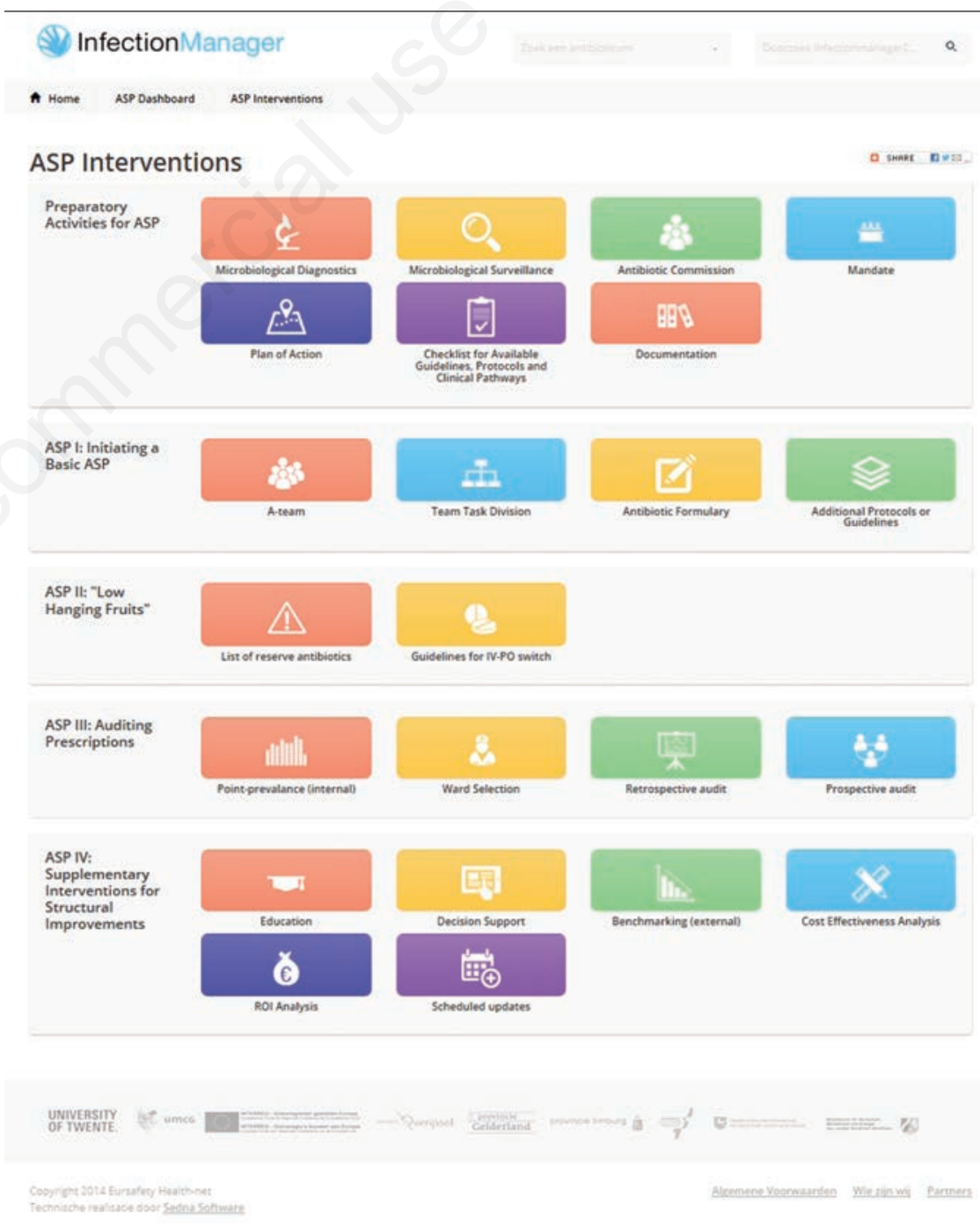

Figure 2. The Maturity model. ${ }^{14}$ 
the improved integration of working with the Prevalence app in their work processes. It enabled them to pause and continue registration at a later point in time, without losing data (as opposed to the prior system).

\section{Conclusions}

In this position paper we have highlighted how technology can be used to contribute to fight antimicrobial resistance (AMR). AMR is a huge wicked problem on a global level, requiring innovative methods and models on a local level to empower our public and professionals to be proactive rather than reactive in a digitalized world. Technology supports the development of an integrated ASP, crossing borders between healthcare and public health. ${ }^{5}$

The CeHRes Roadmap provides the methodology to guide the ASP toolkit development process, providing methods for involving end-users (to create and test prototypes) and stakeholders (to define the added values and conditions for the implementation). The digitalization of information and the options for mobile technology facilitate an integrated and technology based Antimicrobial Stewardship Model. ${ }^{6}$ Furthermore, ubiquitous digital networks offer facilities for continuous web-based education of the public, healthcare providers and infection prevention experts, making new knowledge easily available through eLearning modules. ${ }^{10}$

The methods and lessons learned that are described in this paper are primarily applicable to and relevant for Antimicrobial Stewardship efforts. This is a highly complex field, that deals with highly complex 'wicked' problems. However, we are convinced that this paper may also be of value for other 'wicked' problems, such as the field of zoonoses. ${ }^{5}$

\section{Future work}

The Centers for Disease Control and Prevention highlighted the key elements for
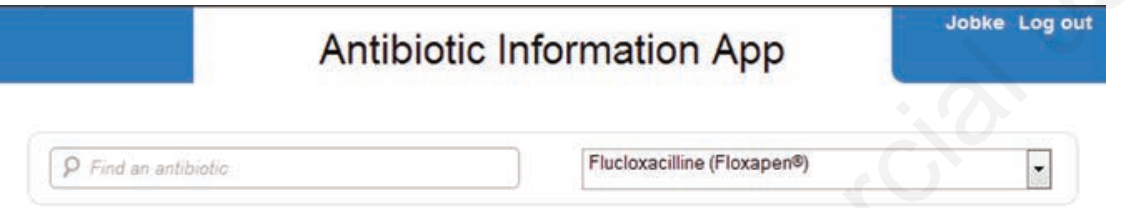

Flucloxacilline (Floxapen $₫$ )

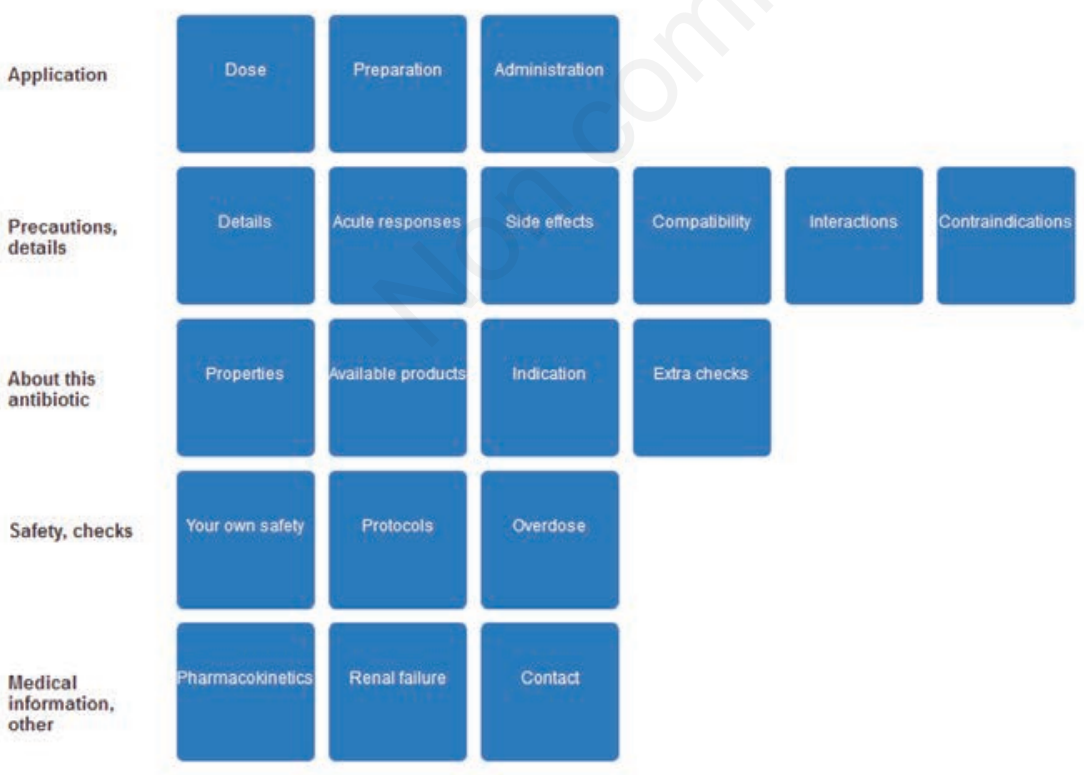

Recent updates

Version

30-01-2013 versie 1.2
ASP, ${ }^{3}$ we think technology can support the realization of the key elements by creating an infrastructure for infection prevention interventions and by continuous surveillance of antimicrobial use.

Automated surveillance systems have already been shown to be sensitive, specific and efficient to prevent the spread of Highly Resistant Micro Organisms (HRMO) and to reduce healthcare-associated infections. ${ }^{21-23}$ However, current surveillance methods lack a systematic approach to detect changes or patterns in events that require acute interventions. Nor is surveillance currently used to predict antimicrobial resistance problems and upcoming outbreaks of diseases due to the lack of advanced analytics. Predictive analytics, using technologies to learn from historical data to make predictions about future health, are needed to enrich the current surveillance systems. Today an extensive growth in the amount of data in healthcare is digitally generated, collected, and stored via mobile and wearable technologies. These technologies provide opportunities to develop predictive, automated decision support systems, for public health and medical care settings. Using advanced algorithms in real-time modus and facilitating risk communication for proactive decision making in the prevention of HRMO outbreaks. We need to ensure there is a human standard in these developments, so that such information is not only available but also accessible and usable for health care work-

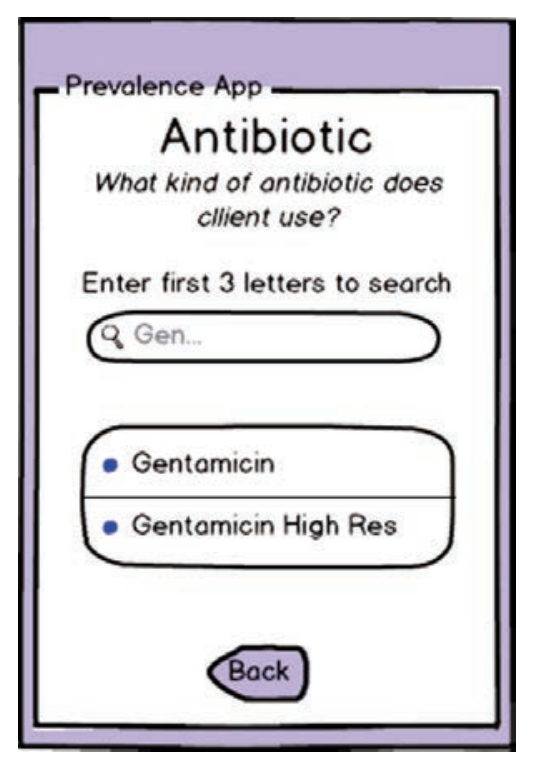

Figure 4. Example of suggestion in a lowfidelity prototype. ${ }^{11}$
Figure 3. The Antibiotic Information app to support nurses in Antimicrobial Stewardship Programs. ${ }^{17}$ 
ers, patients, citizens, and other stakeholders during their daily life or work. In current AMR projects (i.e., INTERREG Va Health$i$-care, eZoon ZonMw) we use the ASP toolkit to develop an eLearning environment and a web-based early warning decision support system to educate professionals and public, to reduce AMR.

\section{References}

1. MacDougall C, Polk RE. Antimicrobial stewardship programs in health care systems. Clin Microbiol Rev 2005;18:638-56.

2. IRIN. When the drugs don't work. 2016. Available from: https://www.irinnews.org/news/2016/09/22/whendrugs-don $\% \mathrm{E} 2 \% 80 \% 99$ t-work

3. Centers for Disease Control and Prevention. Core elements of hospital antibiotic stewardship programs. Atlanta: CDC, 2014.

4. Centers for Disease Control and Prevention. Antibiotic resistance threats in the United States, 2013. Atlanta: CDC; 2015.

5. van Woezik AF, Braakman-Jansen LM, Kulyk O, et al. Tackling wicked problems in infection prevention and control: a guideline for co-creation with stakeholders. Antimicrob Resist Infect Control 2016;5:1.

6. Dik J-WH, Poelman R, Friedrich AW, et al. An integrated stewardship model: antimicrobial, infection prevention and diagnostic (AID). Future Microbiol 2016;11:93-102.

7. van Limburg M, Wentzel J, Sanderman R, van Gemert-Pijnen L. Business modeling to implement an eHealth portal for infection control: a reflection on co-creation with stakeholders. JMIR Res Prot 2015;4.
8. van Gemert-Pijnen JE, Nijland N, van Limburg M, et al. A holistic framework to improve the uptake and impact of eHealth technologies. J Med Internet Res 2011;13:e111.

9. van Gemert-Pijnen J, Peters O, Ossebaard HC. Improving eHealth. Den Haag: Eleven International Publishing; 2013.

10. Kelders S, Sieverink F, Kip H, GemertPijnen Lv. eHealth: combining psychology, technology and health. FutureLearn; 2016. Available from: https://www.futurelearn.com/courses/e health.

11. Beerlage-de Jong N, EikelenboomBoskamp A, Voss A, Sanderman R. Combining user-centered design with the persuasive systems design model; the development process of a webbased registration and monitoring system for healthcare-associated infections in nursing homes. Int $\mathrm{J}$ Adv Life Sci 2014;6:262-71.

12. Oinas-Kukkonen $H$, Harjumaa $M$. Persuasive systems design: key issues, process model, and system features. Commun Assoc Informat Syst 2009;24:28.

13. Beerlage-de Jong N, Wentzel MJ, Dik JW, et al. Opening the black box of clinical decision support systems for antimicrobial stewardship - a scoping review. Int J Medical Informatics.

14. Limburg AHM. Implementing antibiotic stewardship: involving stakeholders in eHealth: University of Twente; 2016.

15. van Limburg M, Sinha B, Lo-Ten-Foe JR, van Gemert-Pijnen JE. Evaluation of early implementations of antibiotic stewardship program initiatives in nine Dutch hospitals. Antimicrob Resist Infect Control. 2014;3:33.

16. Limburg MV, Ossebaard HC, Wentzel J, Gemert-Pijnen LV, eds. Co-creating with stakeholders: ideating eHealth applications to support antibiotic stewardship in hospitals. eTELEMED 2016: The Eighth International Conference on eHealth, Telemedicine, and Social Medicine 2016; Venice, Italy: IARIA.

17. Wentzel J, van Velsen L, van Limburg $\mathrm{M}$, et al. Participatory eHealth development to support nurses in antimicrobial stewardship. BMC Med Inform Decis Mak 2014;14:1-12.

18. Wentzel MJ. Keeping an eye on the context: participatory development of eHealth to support clinical practice: Universiteit Twente; 2015.

19. Rosenfeld L, Morville P. Information architecture for the World Wide Web: Designing large-scale web sites.: O'Reilly Media, Inc.; 2002.

20. Chaboksawar T. Evaluatie van de gebruiksvriendelijkheid en persuasiviteit van een web-based applicatie Een vergelijking tussen het oude en het nieuwe systeem. Enschede: University of Twente; 2014.

21. Streefkerk RH, Borsboom GJ, van der Hoeven CP, et al. Evaluation of an algorithm for electronic surveillance of hospital-acquired infections yielding serial weekly point prevalence scores. Infect Control Hosp Epidemiol 2014;35:88890.

22. Streefkerk RH, Moorman PW, Parlevliet GA, et al. An automated algorithm to preselect patients to be assessed individually in point prevalence surveys for hospital-acquired infections in surgery. Infect Control Hosp Epidemiol 2014;35:886-7.

23. van Mourik MS, Troelstra A, van Solinge WW, et al. Automated surveillance for healthcare-associated infections: opportunities for improvement. Clin Infect Dis 2013;57:85-93. 